proteases. Such a weakly structured region, they hazard, might respond to an applied tension by uncoiling. This extended form will resist any further tension, and might be expected to annihilate it by a condensation process. Burke et al. interpret in these terms the biphasic change of tension in a muscle subjected to a rapid change in length, which was recently described by Huxley and Simmons. They have attempted to induce a change in the tendency of the rod to distort by addition of contractionlinked ligands, such as ATP, ADP, and calcium and magnesium ions, though without success.

The indications are that the other $\alpha$ helical muscle proteins, in particular the molluscan paramyosins, which have no globular heads, are very similar in most important structural respects to the myosin rod, and Halsey and Harrington (ibid., 693) have undertaken a parallel study on clam paramyosin. As with myosin rods, tryptic hydrolysis displays a very rapid initial phase in which a labile region is destroyed. A "light meromyosin" is generated, which melts thermally in a single transition, corresponding to the higher of two transitions observed in the melting of the intact paramyosin. The amino-acid compositions of the two parts of the molecule differ and that of the surviving rod has a higher computed $\alpha$-helix-forming potential (at least by criteria drawn from analysis of globular protein conformations); indeed the composition of the material released in the early stages of trypsin hydrolysis should in these terms be distinctly unfavourable for the stability of $\alpha$-helices. This lends support to the possibility of a region of distorted, or at least weakened, $\alpha$-helix in this molecule. Cowgill in a report last year also showed evidence of two regions in the molecule differing in stability, the stable "light meromyosin" being the $\mathrm{N}$ terminal end.

As to the question of whether and why two heads are in fact better than one, Margossian and Lowey (J. Mol. Biol., 74, 301; 1973) have set out in search of an answer by preparing, by papain treatment under highly selective conditions, followed by ion-exchange chromatography, one-headed myosin, as well as one-headed heavy meromyosin. Properties such as molecular weights and $\alpha$-helix contents coincide nicely with the expected values, and the fragments contain their complements of light chains. The mutilated molecules look exactly as they should in the electron microscope. In the accompanying article (ibid., 313), which has all the attributes of a definitive utterance, Margossian and Lowey compare the functional properties of the one and two-headed species as well as isolated heads with no shaft (subfragment-1). The ATPase turnover of the two-headed protein is, as nearly as it is possible to estimate, twice that of the one-headed derivatives, which indicates the self sufficiency, and to a first approximation independence, of the two heads. The actin-binding stoichiometry, measured with the ultracentrifuge, is one actin subunit per head, in agreement with recent work of Eisenberg and coworkers, and in contradiction of earlier results of Young. A somewhat tortured analysis of the behaviour of unfractionated mixtures of partially inactivated myosins by Toikawa and Morales not long ago led them to the conclusion that both heads of myosin must be operative for superprecipitation with actin. Margossian and Lowey have been able to consign this notion to limbo by showing that their one-headed myosin when mixed with $F$ actin superprecipitates very nicely. Only the time lag is longer, and this may be put down to the greater affinity with which the two-headed species would be expected to interact with the F actin.

The problem of why myosin need have two heads is still unanswered, leaving only some enigmatic fragments of evidence against their precise equivalence. Anybody with a serious interest in the field will want to study the analysis given by Margossian and Lowey of ATPase activity in relation to actin binding, which goes some way towards clarifying a situation obfuscated by stratum upon stratum of hypothesis.

\section{CONSERVATION \\ Galapagos Endemics}

from our Plant Ecology Correspondent IN 1971 Wiggins and Porter published a new Flora of the Galapagos Islands (Stanford University Press, California) which has provided the raw material and the necessary incentive for the initiation of more extensive research into the flora of these islands. Johnson and Raven (Science, 179, 893; 1973) have subjected the data from this Flora to rigorous statistical and phytogeographical analysis.

Only 26 per cent of the Galapagos flora is endemic, a figure which compares poorly with the Hawaiian Islands where more than 90 per cent of the vascular plant species are endemic. The percentage of endemics in the individual floras of the Galapagos Islands is inversely related to the area of the islands, a situation which can be explained by reference to the pattern of

\title{
A Persistent Cell Population in the Thymus
}

THE thymus in the centuries following its first description was regarded as a mysterious organ. Many and bizarre were the functions ascribed to it until the works of Miller and of Good showed that it regulated the development of immunological responsiveness -at least in some species of mammals. Since then it has been revealed that the thymus performs this function by liberation of what have come to be known as $\mathrm{T}$ lymphocytes. Much emphasis has been placed by cellular immunologists on this versatile species of cell and its more productive ally (as far as antibodies are concerned), the B lymphocyte. But, aside from its capacity for producing cells, the thymus itself has retained much of its mystery. It is suspected to emit a hormone, but hardly anything is known of the process of differentiation whereby the organ gives rise to $\mathrm{T}$ cells. In next Wednesday's Nature New Biology (April 4) Elliott deals with heterogeneity of cells within the thymus.

It is usually accepted that after treatment of a mouse with corticosteroids, such as hydrocortisone, there remains in the thymus, after much cell death, a population of steroid-resistant cells. In an earlier report, Elliott et al. (Nature New Biology, 234, 77; 1971) showed that these cells were not $\mathbf{T}$ cells that had come in from outside the organ but were cells that were in the thymus at the time of treatment with corticosteroid.

The method involved the engraftment of a chromosomally-marked but otherwise syngeneic thymus graft into a normal mouse and then, after treatment with hydrocortisone, display of the phytohaemagglutinin (PHA)-responsive cells from the graft in vitro. Elliott now extends this observation and shows that with or without treatment with hydrocortisone most of the cells in a thymus graft at any time after its emplacement, that can be induced to respond to PHA, are cells that are native to the graft.

The thymus is usually thought of as a staging house through which pass stem cells and their mitotically amplified products. In a thymus graft active mitosis of the native cells in the graft itself ceases by about the twentieth day after grafting and yet, as Elliott shows, many weeks later the PHA-responsive cell population revealed in vitro contains many of the native cells which previously would have been assumed to have emigrated to the periphery.

The functional significance of this persistent cell population is not known nor is the morphology of the persistent cell. Immunologists who think of thymocytes as equivalent to peripheral $T$ cells should, however, remember that they are also handling a cell population which never seems to leave the thymus. 